\title{
The function of human epidermal growth factor receptor-3 and its role in tumors (Review)
}

\author{
QIN LI, ZHENYAN YUAN and BANGWEI CAO \\ Department of Oncology, Beijing Friendship Hospital, Capital Medical University, Beijing 100050, P.R. China
}

Received July 18, 2013; Accepted September 6, 2013

DOI: $10.3892 /$ or.2013.2754

\begin{abstract}
Human epidermal growth factor receptor-3 (HER-3) is the third member of the HER family. It was previously considered not to contain tyrosine kinase activity and catalytic activity and the intracellular region of HER-3 could not bind ATP and be auto-phosphorylated. Thus, the clinical value of HER-3 was ignored. Currently, biochemical analysis has confirmed that the kinase domain of HER-3 is a specific allosteric activator; it acts as a functional activator to activate the recipient kinase (HER-1, HER-2, HER-4). With the in-depth knowledge of its structure and function, studies on the relationship of HER-3 and human tumors are rapidly increasing. HER-3 is closely related to tumorigenesis, progression and metastasis. HER-3 is involved in resistance to targeted therapy, and may serve as a new therapeutic target. The expression of HER-3 helps to predict prognosis and treatment efficacy. HER-3 has become a focus of concern in the HER family and has gained significant attention in the search for cancer treatment.
\end{abstract}

\section{Contents}

1. Introduction

2. In-depth understanding of the structure and function of HER-3

3. The close relationship between HER-3 and human tumor

4. Conclusion

\section{Introduction}

The human epidermal growth factor receptor (HER or ErbB) belongs to the tyrosine kinase receptor superfamily. It includes

Correspondence to: Dr Bangwei Cao, Department of Oncology, Beijing Friendship Hospital, Capital Medical University, 95 Yong An Road, Xicheng District, Beijing 100050, P.R. China

E-mail: oncologychina@163.com

Key words: human epidermal growth factor receptor-3, ErbB3, human epidermal growth factor, tumor
4 highly homologous members, HER-1 (ErbB1), HER-2 (ErbB2), HER-3 (ErbB3) and HER-4 (ErbB4). The distinguishing characteristics of the HER family are interdependent and functional complementation between members. After the ligands bind to the receptor, it promotes the formation of HER/ErbB receptor homodimer or heterodimer which leads to activation of the tyrosine kinase domain $(1,2)$ and downstream signaling pathways $(3,4)$. Signal transduction networks control cellular activities such as gene expression, mitosis, cell differentiation, cell proliferation, cell survival and apoptosis $(1,5)$.

HER-3 is a distinctive member of the HER family as its kinase domain lacks certain residues that are known to be essential for catalytic activity in other kinases. The function of HER-3 was previously thought to be entirely dependent on other members of the family, the role of HER-3 was considered to be passive and the clinical value of HER-3 was greatly underestimated. Currently, biochemical analysis has confirmed that the kinase domain of HER-3 is a specific allosteric activator, it acts as a functional activator to activate the recipient kinase. Accompanied by an in-depth understanding of the structure and function of HER-3, recent studies have also found HER-3 is involved in the tumorigenesis, progression, new target exploration, target therapy resistance of several types of cancer. In the critical search of a cure for cancer, HER-3 provides insight into the better understanding of tumors and targeted therapy.

\section{In-depth understanding of the structure and function of HER-3}

HER-3 was initially isolated by MH Kraus in 1989. The gene of HER-3 is located on chromosome $12 \mathrm{q} 13$, and its $6.2 \mathrm{~kb}$ transcript is expressed in normal epithelial tissues (6). Subsequently, HER-3 cDNA was isolated from human tumor cell lines (7). HER-3 possesses $40-50 \%$ sequence homology with HER-1 and 40-45\% homology with HER-2 (7-9). The structure of HER-3 is typical in receptor tyrosine kinase family (Fig. 1). It includes an extracellular domain (ECD) with 612 amino acid residues, a transmembrane helix domain with 32 hydrophobic amino acids, and an intracellular tyrosine kinase domain (TKD) with 677 amino acids (7). Determination of the 2.6-angstrom crystal structure of the entire extracellular region of HER-3 revealed 4 type I insulin-like growth factor receptor homologous domains, 2 specific ligand binding flanking regions (district I and III) and 2 cysteine-rich regions (district II and IV). The interaction between district II 
and IV limits the relative direction of ligand binding domain and provides a structural basis for understanding the varied affinity and conformational changes of HER-3 upon ligand binding (10). The transmembrane region confers receptor internalization and ligand-dependent calcium influx (11). ErbB binding protein 1 (EBP-1) interacts with HER-3 and prevents the premature formation of dimers, which prevents inappropriate activation of molecular partners by HER-3 (12). The intracellular domain is a continuation of the transmembrane region and has a conserved ATP binding site (Gly-Xaa-GlyXaa-Xaa-Gly-Xaa-Lys) that shares homology with other members of the tyrosine kinase family. The intracellular region is divided into a juxtamembrane region, a kinase domain and $\mathrm{C}$-terminal tail. The juxtamembrane region is divided into N-terminal [juxtamembrane-A, (JM-A)] and C-terminal [juxtamembrane-B, (JM-B)] (13). The kinase domain includes $\mathrm{N}$-lobe, helix $\alpha \mathrm{C}$, activation loop and C-lobe (13). The analysis of HER-3 dimers crystal structure shows that recipient protein kinase (HER-1, HER-2 and HER-4) interacts with the JM-A region of HER-3 via a conserved amino acid sequence, and the JM-B region of recipient protein kinase interacts with C-lobe of HER-3 by forming a stabilizing latch (14). C-lobe of HER-3 can combine and activate other members of the HER family, which is consistent with the role of HER-3 as a functional activator but not a recipient kinase. Helix $\alpha \mathrm{C}$ of the kinase domain anchors activator kinase domain to recipient kinase domain (13). In general, HER-3 is very similar to inactivated HER-1/HER-4. Conformational changes in helix $\alpha \mathrm{C}$ sequence are highly important and may explain the difference in function of HER-3 compared with other members of the family. For example, Leu736 in helix $\alpha \mathrm{C}$ of HER-1 is replaced by Thr738 in HER-3, and this change stabilizes the inactivation state of HER-3. Ile735 in hydrophobic core of HER-1 is replaced by Val737 in HER-3, and this change weakens the ability of HER-3 to form hydrophobic subunits. The structure of HER-3 is similar to that of an integrate kinase, but locked in an inactive conformation similar to that of Src/CDK. When the HER-3 sequence is activated, helix $\alpha \mathrm{C}$ turns to the active site, the activation ring center is opened and bound the substrate peptide. There are 14 tyrosine residues in HER-3 C-terminal signal tail, including six PI3K binding sites that have been confirmed through phosphorylation, which mediate interactions between three regulatory subunits of PI3K and lead to activation of downstream AKT signaling pathways (15).

At present, our understanding of the function of HER-3 is very limited. In the 1990s, the kinase activity of HER-3 was not detected by recombinant protein technique. Therefore, researchers believed that the kinase domain of HER-3 may be non-functional $(16,17)$. HER-3 kinase domain lacks several key residues required for catalytic activity, such as Asp813 which is present in HER-1; thus HER-3 was thought not to contain tyrosine kinase activity and catalytic activity. The intracellular region of HER-3 does not bind ATP and is not auto-phosphorylated $(18,19)$. HER-3 was considered to be functional merely as a signaling substrate for other HER members, similar to the function of insulin receptors, IRS1 and IRS2. However, Kornev and Taylor's (20) study in 2009 demonstrated that HER-3 was not completely inactive; its activity was very low and was not comparable to that of HER-1, but the kinase activity of HER-3 was sufficient to mediate auto-phosphorylation of its intracellular region. Shi et al (21), using molecular mechanics simulation in 2010, revealed that the phosphorylation catalyzed by HER-3 was mediated via the 'inactive-like' structure rather than the conserved catalytic subunit, suggesting that the cytoplasmic region of HER-3 within the receptor dimers was capable of binding ATP and promoting auto-phosphorylation. Jura et al (13) confirmed, using biochemical analysis in 2009, that the kinase domain of HER-3 was a specific allosteric activator to activate the recipient protein kinase. Although these studies challenged the traditional understanding of HER-3 function, the exact mechanism is not fully understood and further studies should be carried out. The function of HER-3 should be re-inspected, the role of HER-3 in cell signaling transduction and human cancer is becoming increasingly important. Findings with regard to the function of HER-3 may provide insight into the pathogenesis and therapy of human cancer.

Following ligand binding, HER-3 forms a receptor dimer via a unique mechanism by which monomeric inactive state changes to active state upon homo- or heterodimerization and the tyrosine kinase and its downstream signaling pathways are activated (22). HER-3 interacts with other members of the HER family. First, HER-3 interacts with HER-1 directly. EGF can activate the tyrosine kinase of HER-1, and can also cross-activate HER-3 at the same time (22). Tyrosine kinase inhibitor (TKI) blocks downstream signaling pathways by inhibiting the interaction of HER-1 and HER-3 (23). Second, HER-3 interacts with HER-2 directly. Heregulin (HRG) ligand binding causes prolonged activation of HER-3, but this process is strongly dependent on HER-2 expression. In the HER family, HER-3 mainly interacts with HER-2. Since HER-2 lacks ligand-binding activity and HER-3 lacks catalytic kinase activity, both the receptors are functionally interdependent. The relationship between HER-2 and HER-3 has been described as 'deaf' and 'dumb' $(18,24,25)$. The cooperation between HER-2 and HER-3 is unique, but the underlying molecular mechanism is poorly understood. Third, HER-3 and HER-4 can form heterodimer, but few studies have explored the relationship between them (26).

There are 2 relevant downstream signaling pathways of HER-3 (Fig. 2). The first one is the phosphatidylinositol 3-kinase $(\mathrm{PI} 3 \mathrm{~K}) / 3$-phosphoinositide-dependent protein kinase (PDK) 1/protein kinase B (AKT) pathway. Activation of PI3K is induced by the formation of dimer between HER-3 and HER-1/2. PI3K is a dimeric protein kinase composed of P110 catalytic subunit and P85 regulatory subunit (27). The P85 subunit binds to HER-specific anchor sites via its $\mathrm{SH} 2$ domain, and the P110 subunit catalyzes the phosphorylation of phosphatidylinositol 4,5-bisphosphate (PIP2) to 3,4,5-triphosphate phosphatidylinositol (PIP3). The level of PIP3 is regulated by phosphatase and tensin homologue deleted on chromosome 10 (PTEN) (28). PI3K accumulates PDK1/AKT in the cell membrane and activates it via phosphorylation, thus stimulating downstream signaling. The PI3K pathway regulates cell growth, cell apoptosis, tumor cell invasion, as well as metastasis and chemotherapy resistance. The second pathway is the Ras/Raf/MEK/mitogen-activated protein kinase (MAPK) pathway. Activation of HER-3 and subsequent phosphorylation of tyrosine kinase induce Grb2-SOS complex binding to phosphorylation anchor sites. Then, the three-dimensional 


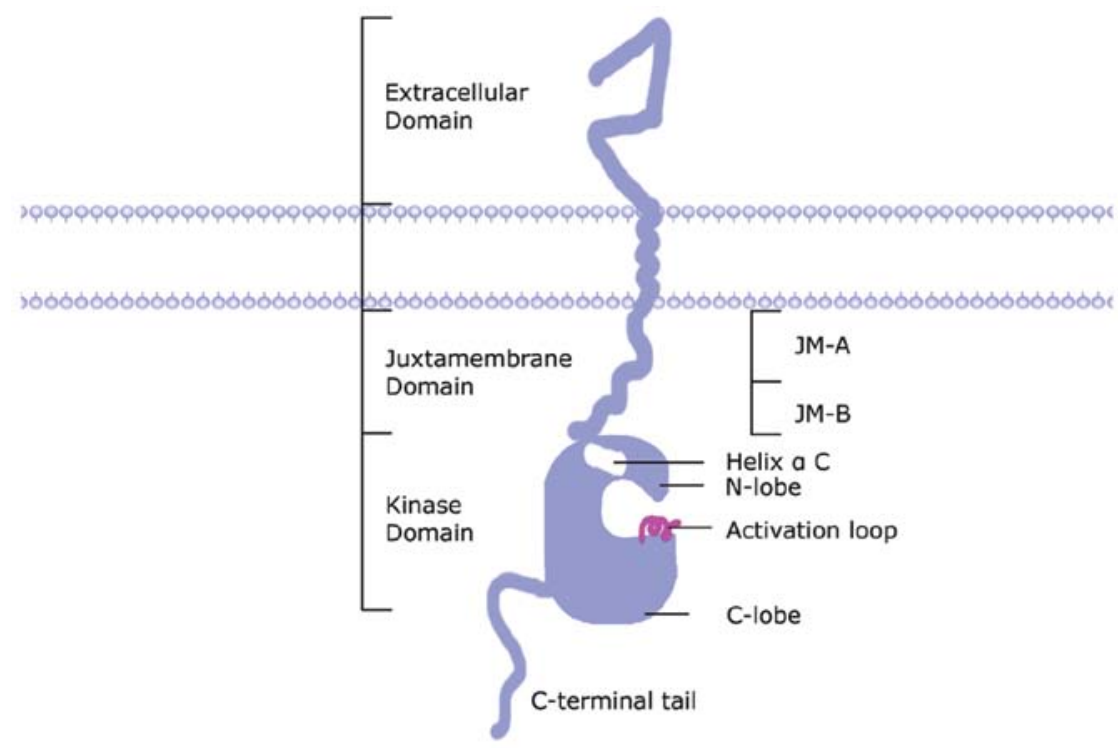

Figure 1. HER-3 structure diagram. The structure of HER-3 includes an extracellular ligand binding domain, a transmembrane helix domain and an intracellular TKD. The intracellular region is divided into a juxtamembrane region, a kinase domain and C-terminal tail. The juxtamembrane region is divided into JM-A and JM-B. The kinase domain includes N-lobe, helix $\alpha$ C, activation loop and C-lobe. HER-3, human epidermal growth factor receptor-3; TKD, tyrosine kinase domain. JM-A, juxtamembrane-A; JM-B, juxtamembrane-B.

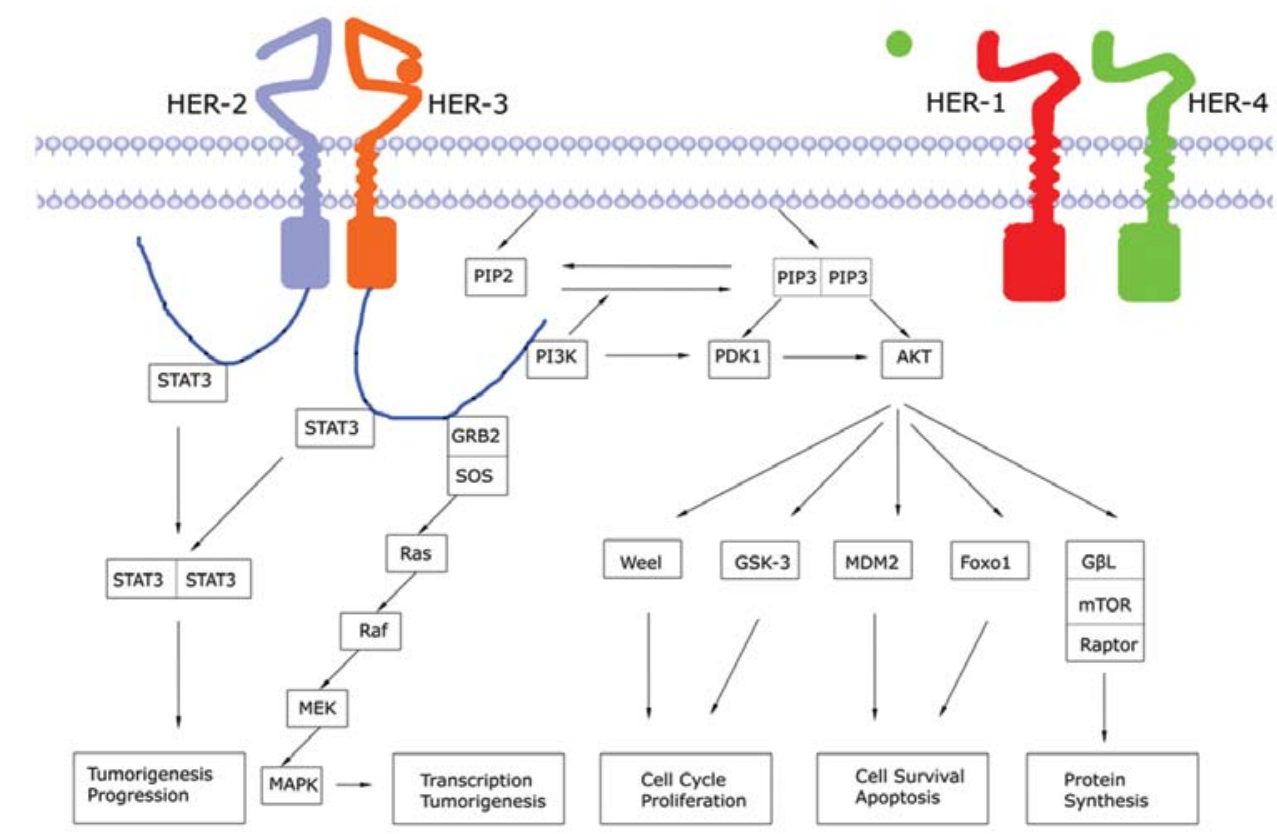

Figure 2. HER-3 and its downstream signaling pathways. The downstream signaling pathways include the PI3K/PDK1/AKT and the Ras/Raf/MEK/MAPK pathway. HER-3, human epidermal growth factor receptor-3; MAPK, mitogen-activated protein kinase.

structure of SOS is altered which enables the formation of Ras-GTP from aggregated Ras-GDP $(29,30)$, leading to the activation of Raf, MEK and MAPK $(31,32)$. Activated MAPK transduces extracellular stimuli into the cell to regulate transcription factors in the nucleus and induces cell migration and proliferation (33).

\section{The close relationship between HER-3 and human tumor}

The studies on HER-1 and HER-2 in tumor targeted therapy and efficacy prediction have developed. For example, small molecule HER-1 TKI, gefitinib, has been used in the first-line treatment of advanced non-small cell lung cancer (NSCLC) with HER-1 mutation (34). Anti-HER-1 monoclonal antibody, cetuximab, has been used in targeted therapy for head and neck squamous cell carcinoma, colorectal cancer and advanced NSCLC (35-37). Anti-HER-2 monoclonal antibody, trastuzumab, has been used in targeted therapy for HER-2 positive advanced breast cancer and gastric cancer $(38,39)$. In view of the significant contribution of HER-1 and HER-2, researchers began looking into the role of HER-3. With the new understanding of the structure and function of HER-3, studies on the 
relationship of HER-3 with tumorigenesis, progression, new target exploration, target therapy resistance, prognosis and efficacy prediction are increasing. Some studies have confirmed that HER-3 plays an important role in the occurrence and progression of lung cancer, breast cancer, colorectal cancer as well as other types of cancer (26,40-42). The HER-3/PI3K/AKT signal pathway plays a key role in the target therapy resistance of NSCLC, breast cancer, head and neck squamous cell carcinoma, prostate cancer and hepatocellular carcinoma and other types of cancer (43-48). Inhibition of HER-3 and HER-1/HER-2 is very important for tumor treatment $(49,50)$ and it may be a new therapeutic target. HER-3 is also beneficial in predicting the prognosis and treatment efficacy. In the search for tumor treatment, HER-3 has become a focus of concern in the HER family. The following describes the role of HER-3 in different tumors.

Overexpression of HER-3 in NSCLC cell lines accelerated growth and metastasis of tumor cells, and promoted tumorigenicity of allografts in a kinase-dependent manner. By contrast, downregulation of HER-3 inhibited proliferation and migration of tumor cells, tumor growth and metastasis in vivo. HER-3 silencing inhibited tumor cell growth by reducing DNA synthesis and caspase-8-mediated apoptosis, and tumor cell migration by increasing accumulation of focal adhesion components (40). Lung adenocarcinoma cells were markedly suppressed in culture by siRNAs to the receptor HER-3 or its downstream signaling partner AKT2 (51). The above studies suggest that HER-3 and its downstream signaling pathway play a crucial role in occurrence and metastasis of lung cancer. HER-1 TKI such as gefitinib/erlotinib is very effective treatment for NSCLC with HER-1 mutation, but the emergence of drug resistance is difficult to overcome. The sensitivity of HER-1 TKI is associated with inhibition of the HER-3/PI3K/ AKT signaling pathway, i.e., this pathway will lead to TKI resistance if not effectively inhibited (43). Gefitinib temporarily inhibits HER-3/PI3K/AKT signaling, but in the process of subsequently sustained inhibition of HER-1 and HER-2, recovery of HER-3 activity and reactivation of the PI3K/ AKT pathway will lead to drug resistance. Downregulation of HER-3 led to reduction of AKT phosphorylation level and growth inhibition in an NSCLC mutant cell line that was sensitive to gefitinib. However, downregulation of HER-3 did not alter the activity of AKT in resistant tumor cell lines, suggesting that the separation of HER-3 from downstream AKT signaling pathway was one of the important aspects of the drug resistance (52). HER-3 and PI3K/AKT pathways may enable tumor cells to escape TKI inhibition through a compensatory offset of the equilibrium between HER-3 phosphorylation and dephosphorylation (43). Amplification of MET proto-oncogene promoted HER-3-dependent PI3K activation and led to drug resistance in gefitinib-sensitive lung cancer cell lines, inhibition of MET proto-oncogene restored the sensitivity to gefitinib (48). In addition to HER-1 mutation, a second mutation, T790M, is also associated with acquired drug resistance. Introduction of exogenous HER-1 with T790M mutation effectively blocked gefitinib activity and maintained HER-3/PI3K/AKT signal activation in lung cancer cells (53). In addition, oncogenic mutation of PIK3CA, p110 $\alpha$ E545K, activated PI3K signaling and eliminated gefitinib-induced apoptosis (54). These studies demonstrated that HER-3 and/ or PI3K/AKT signal may be intermediate links of acquired gefitinib resistance. While irreversible tyrosine kinase agents inhibited HER-1 in resistant cells, they re-inhibited HER-3 and PI3K signaling at the same time, which further highlighted the central role of HER-3 in adjusting drug sensitivity or resistance (55). miR-22 in lung cancer cell lines played a good antitumor effect through inhibition of HER-3 transcriptional regulation (56). Thus, HER-3 is a potential therapeutic target and simultaneous inhibition of HER-3 and HER-1 will bring considerable benefit to the clinic. Due to the complexity of signaling networks, whether the HER-3/PI3K/AKT pathway alone can induce TKI resistance in NSCLC merits further study. The positive expression rates of HER-3 in NSCLC are 18-67\%. Study results on the response to TKI, survival and prognosis of patients with HER-3 high expression are inconsistent. A study including 192 surgically removed NSCLC cases showed the expression of HER-3mRNA was higher in patients with highly-differentiated, adenocarcinoma, mutant HER-1 than in patients with poorly-differentiated, nonadenocarcinoma, wild-type HER-1, and the expression was higher in females than in males (57). Large sample studies and more uniform and accurate research methods are required to further clarify whether high HER-3 expression is an indicator of TKI benefit.

The role of the HER family in tumorigenesis is most aptly understood in breast cancer subtypes with HER-2 gene amplification. HER-3 is required for HER-2-induced preneoplastic changes to breast tumor formation (58). HER-2 must dimerize with HER-3 to promote breast cancer cell proliferation; deletion of HER-3 in HER-2 positive breast cancer cell lines produces strong anti-proliferative effects (59). HER-2/ HER-3 heterodimer is the most powerful carcinogenic unit, the phosphorylation state of HER-3 is critical for HER-2 positive breast cancer cell motility and metastasis (41). A significant increase of HER-3 phosphorylation in HER-2 positive breast cancer was accompanied by activation of downstream signaling pathways, and knockdown of HER-3 was always accompanied by tumor shrinkage in vitro (60). Dephosphorylation of HER-3 and decoupling with PI3K lead to downregulation of AKT signaling and it is directly related to the anti-proliferative effect of trastuzumab. HER-3-specific affibody molecules (Z05416, Z05417) blocked cancer cell growth by inhibiting HRG-induced HER-3 phosphorylation in the MCF-7 and SKBR-3 breast cancer cell lines (61). The above studies clarify that the phosphorylation state of HER-3 and downstream signaling play a central role in the occurrence of HER-2 positive breast cancer, and HER-3 may be a drug target. Since the process of TKI inhibiting HER-2/HER-3 transphosphorylation and PI3K/AKT signaling pathway activation in HER-2 positive breast cancer are transient, the antitumor efficacy of TKI is weakened (43). The kinase function of HER-2 is essential during tumorigenesis (62), therefore, inhibition of the catalytic kinase activity is a key mechanism for anticancer drugs. The buffering effect of incomplete inhibition of HER-2 kinase activity by HER-3 may be a highly important mechanism by which HER-2 positive breast cancer cells escape TKI treatment $(43,63)$. miR-205 downregulates HER-3 and recovers the sensitivity to TKI in human breast cancer cells (64). pHER-3 upregulation in a fulvestrant resistant cell line was mostly accompanied by an 
increase of pAKT activity. However, highly specific inhibition by HER-3 antibody (A5) significantly downregulated pHER-3 without affecting downstream ERK/AKT phosphorylation, suggesting that the resistant cells may produce endogenous ligand that reactivated pHER-3. Exogenous ligands binding to HER-3 affect AKT downstream signals, but the underlying mechanism remains unclear (47). Bispecific antibody (MM-111) formed trimer with HER-2/HER-3, which effectively inhibited proliferation of HER-3 and HER-2 positive tumor cells (50). A new selective PI3K inhibitor (GDC-0941) combined with trastuzumab and pertuzumab inhibited the growth of tumor cells, and led to morphological changes of gland cells and inhibition of the HER-3/PI3K/AKT signal pathway (65). GDC-0941 is also effective for the treatment of trastuzumab-resistant tumor cells (66). XL147, which is also a PI3K inhibitor, inhibits tumor cell growth in a dose-dependent manner. In HER-2 positive breast cancer cells, knockdown of HER-3 by siRNA enhanced the effect of XL147 (67). Thus, multi-target treatment provides an increase of clinical benefit for patients with breast cancer, inhibition of HER-2, HER-3 and PI $3 \mathrm{~K}$ simultaneously may be the future treatment direction of HER-2 positive breast cancer. The positive expression rates of HER-3 in breast cancer are $18-75 \%$. The expression of HER-3 was positively correlated with high organizational classification and lymphatic vessel invasion, suggesting it was significantly associated with tumor progression and metastasis, and may serve as a useful prognostic biomarker (68). Tissue microarray analysis demonstrated that normal expression of HER-1/HER-2 and overexpression of HER-3 in invasive breast cancer indicated poor prognosis $(69,77)$. Multivariate analysis showed that HER-2 and HER-3 were independent prognostic markers, while clustering analysis showed that coexpression of HER-1 and HER-3 suggested poor prognosis, with a 10-year survival rate of $42 \%$ (70). However, in HER-2 negative/low expression cases or HER-2 positive breast cancer patients treated with trastuzumab, there was no correlation between the expression of HRG, HER-3 and survival or known clinical prognostic factors $(71,72)$.

There is almost no HER-3 expression in normal colon tissue, but the positive expression rates of HER-3 in colorectal cancer tissue are 50-89\%. Knockdown of HER-3 by siRNA in colon cancer cell lines was accompanied by absence of HER-4 expression and elevation of tumor cell apoptosis; HER-3/HER-4 heterodimer may be one of the precipitating factors of colon cancer (26). HRG expression was detected in colon metastatic liver cancer cells, knockdown of integrin $\alpha \mathrm{v}$ and HER-3 by siRNA significantly inhibited HRG-induced tumor cell migration as well as liver metastasis in vivo, marked phosphorylation of AKT was found in the process, cell migration was suppressed by specific inhibitors of PI3K. The study indicated that HRG/HER-3/PI3K/AKT may participate in colon cancer liver metastasis (73). The median progression-free survival time and the median overall survival time of the patients with wild-type K-RAS advanced colorectal cancer receiving cetuximab combining irinotecan treatment in the HER-3 negative group were significantly higher than in the HER-3 positive group, suggesting that HER-3 may be a predictor of cetuximab efficacy in patients with wild-type K-RAS advanced colorectal cancer (74). Comprehensive analysis of HER-3 and K-RAS may aid in identifying the most appropriate colorectal cancer patients for cetuximab treatment and may provide an effective treatment strategy. Studies on HER-3 and other digestive system tumors are limited. The positive expression rate of HER-3 in gastric cancer is $13.7 \%$, and it correlates with late stage and poor prognosis (75). DARPP-32 promotes resistance of gastric cancer cells to gefitinib by stimulating interaction between HER-1 and HER-3 and activating PI3K/AKT signaling (76). The results of the ToGA trial are encouraging; it is expected that routine detection of HER-2 will be included in the diagnosis of advanced gastric cancer $(39,77)$. The trial investigates whether HER-3 will be of value for guiding the treatment of gastric cancer. HER-1/2 expression is absent in normal pancreatic tissue, but HER-3/4 are expressed (78). The positive expression rates of HER-3 in pancreatic cancer tissues are $27-47 \%$. Generally, HER-3 positive was prone to cause targeted therapy drug resistance, but HER-3 increases the sensitivity of pancreatic cancer cells to erlotinib. Knockdown of HER-3 in erlotinib sensitive pancreatic cancer cell lines resulted in AKT level reduction and pancreatic cancer cell proliferation, suggesting that HER-3 may be a sensitive biomarker for erlotinib in pancreatic cancer (79). The median survival time in HER-3 overexpression patients with resectable pancreatic cancer was 37.2 months, but in HER-3-negative patients it was 58.6 months, therefore, HER-3 overexpression may be an independent indicator of poor prognosis for patients with curatively resected pancreatic cancer (80). A study found sHER-3 (isomers) was more accurate than AFP in identifying early liver cancer from chronic hepatitis; the plasma high-level was closely related to portal venous invasion and extrahepatic metastasis (81). HER-3 restricted cell response to sorafenib or IGF1R inhibitor in hepatocellular carcinoma cells $(46,82)$.

HER-3 is activated in multiple ovarian cancer cell lines. Activation of NRG1/HER-3 autocrine loop pathway promotes the proliferation of human ovarian cancer cells. In the mouse xenograft model, deletion of HER-3 inhibited proliferation of OVCAR8 cells and slowed down tumor progression, suggesting that HER-3 and/or NRG1 play a key role in the pathogenesis of ovarian cancer and are potential therapeutic targets for advanced ovarian cancer (83). The positive expression rates of HER-3 in ovarian cancer are 3-53\%, and some studies reported that HER-3 expression was negatively correlated to overall survival $(84,85)$. Regardless of whether the corresponding ligand NRG existed or not, HER-3 promoted prostate cancer cells mobile in vitro and tumor formation in vivo (86). HER-2/HER-3 heterodimer promoted aberrant activation of androgen and led to the formation of hormoneresistant prostate cancer (45). Further studies are required to elucidate the role of HER-3 and other HER members in reproductive system tumors.

High expression of HER-3 and absence of HER-2 expression in melanoma indicated that HER-3 may be an allosteric activator of HER-1 or HER-4. Disorders of NRG1/HER-3 and HRG/HER-3 signaling are correlated with development and metastasis of melanoma $(87,88)$. Anti-human HER-3 monoclonal antibody promoted HER-3 receptor internalization and degradation, and inhibited growth and migration of human melanoma cells (89). The pan-HER receptor TKI (canertinib) inhibits HER-1, HER-2 and HER-3 receptor phosphorylation and promotes apoptosis of malignant melanoma in vitro; it also displays antitumor activity in vivo (90). 


\section{Conclusion}

With protein crystallization, molecular biology has begun to reveal the structure of HER-3. At the same time, the kinase domain of HER-3 acting as a functional activator to activate the recipient kinase was confirmed. Insights into the activation mechanism of the HER family were also gradually elucidated. In the critical search of a cure for cancer, HER-3, as a member recognized step by step in the HER family, seems to provide some insight into tumor therapy. Although people have known about HER-3 for several years, the value of HER-3 was formerly ignored. Currently, research results demonstrate that HER-3 is closely related to tumorigenesis, progression and metastasis, which helps to clarify the mechanisms of tumor biological behavior. HER-3 is involved in targeted therapy resistance and may be a new therapeutic target. A further in-depth understanding of HER-3 will play a fueling role in HER-3 associated targeted therapies. Analyzing the relationship between the expression of HER-3 and the effects of targeted therapy may help to identify the most appropriate patient sub-groups for HER-3 targeted treatment. In the search for a breakthrough in cancer treatment, HER-3 has become an emerging protagonist in the HER family.

\section{Acknowledgements}

The authors thank Professor Xinjun Lv (Chinese Center for Disease Control and Prevention) for direction on review and Professor Enyun Shen (Shandong Provincial Academy of Medical Sciences) for the helpful discussion. This study was supported by grants from the Beijing Municipal '215' Highlevel Health Person Foundation Project (2011-2014 to B.C.), the Beijing Municipal 'Ten, Hundred, Thousand' Person Foundation Project (2011-2013 to B.C.), and the Capital Medical University Sciences-Clinical Research Cooperation Foundation (2011-2012 to B.C.).

\section{References}

1. Olayioye MA, Neve RM, Lane HA and Hynes NE: The ErbB signaling network: receptor heterodimerization in development and cancer. EMBO J 19: 3159-3167, 2000.

2. Schlessinger J: Cell signaling by receptor tyrosine kinases. Cell 103: 211-225, 2000.

3. Marmor MD, Skaria KB and Yarden Y: Signal transduction and oncogenesis by ErbB/HER receptors. Int J Radiat Oncol Biol Phys 58: 903-913, 2004.

4. Shepard HM, Brdlik CM and Schreiber H: Signal integration: a framework for understanding the efficacy of therapeutics targeting the human EGFR family. J Clin Invest 118: 3574-3581, 2008.

5. Yarden Y and Sliwkowski MX: Untangling the ErbB signalling network. Nat Rev Mol Cell Biol 2: 127-137, 2001.

6. Kraus MH, Issing W, Miki T, Popescu NC and Aaronson SA: Isolation and characterization of ERBB3, a third member of the ERBB/epidermal growth factor receptor family: evidence for overexpression in a subset of human mammary tumors. Proc Natl Acad Sci USA 86: 9193-9197, 1989.

7. Plowman GD, Whitney GS, Neubauer MG, et al: Molecular cloning and expression of an additional epidermal growth factor receptor-related gene. Proc Natl Acad Sci USA 87: 4905-4909, 1990.

8. Ullrich A, Coussens L, Hayflick JS, et al: Human epidermal growth factor receptor cDNA sequence and aberrant expression of the amplified gene in A431 epidermoid carcinoma cells. Nature 309: 418-425, 1984.
9. Coussens L, Yang-Feng TL, Liao YC, et al: Tyrosine kinase receptor with extensive homology to EGF receptor shares chromosomal location with neu oncogene. Science 230: 1132-1139, 1985.

10. Cho HS and Leahy DJ: Structure of the extracellular region of HER3 reveals an interdomain tether. Science 297: 1330-1333, 2002.

11. Chen WS, Lazar CS, Lund KA, et al: Functional independence of the epidermal growth factor receptor from a domain required for ligand-induced internalization and calcium regulation. Cell 59: 33-43, 1989.

12. Yoo J, Wang X, Rishi A, et al: Interaction of the PA2G4 (EBP1) protein with ErbB-3 and regulation of this binding by heregulin. Br J Cancer 82: 683-690, 2000.

13. Jura N, Shan Y, Cao X, Shaw DE and Kuriyan J: Structural analysis of the catalytically inactive kinase domain of the human EGF receptor 3. Proc Natl Acad Sci USA 106: 21608-21613, 2009.

14. Red Brewer M, Choi SH, Alvarado D, et al: The juxtamembrane region of the EGF receptor functions as an activation domain. Mol Cell 34: 641-651, 2009.

15. Jones RB, Gordus A, Krall JA and MacBeath G: A quantitative protein interaction network for the ErbB receptors using protein microarrays. Nature 439: 168-174, 2006.

16. Sierke SL, Cheng K, Kim HH and Koland JG: Biochemical characterization of the protein tyrosine kinase homology domain of the ErbB3 (HER3) receptor protein. Biochem J 322: 757-763, 1997.

17. Prigent SA and Gullick WJ: Identification of c-erbB-3 binding sites for phosphatidylinositol 3'-kinase and SHC using an EGF receptor/c-erbB-3 chimera. EMBO J 13: 2831-2841, 1994.

18. Citri A, Skaria KB and Yarden Y: The deaf and the dumb: the biology of ErbB-2 and ErbB-3. Exp Cell Res 284: 54-65, 2003.

19. Manning G, Whyte DB, Martinez R, Hunter T and Sudarsanam S: The protein kinase complement of the human genome. Science 298: 1912-1934, 2002.

20. Kornev AP and Taylor SS: Pseudokinases: functional insights gleaned from structure. Structure 17: 5-7, 2009.

21. Shi F, Telesco SE, Liu Y, Radhakrishnan R and Lemmon MA: ErbB3/HER3 intracellular domain is competent to bind ATP and catalyze autophosphorylation. Proc Natl Acad Sci USA 107: 7692-7697, 2010.

22. Dawson JP, Berger MB, Lin CC, Schlessinger J, Lemmon MA and Ferguson KM: Epidermal growth factor receptor dimerization and activation require ligand-induced conformational changes in the dimer interface. Mol Cell Biol 25: 7734-7742, 2005.

23. Carrasco-García E, Saceda M, Grasso S, et al: Small tyrosine kinase inhibitors interrupt EGFR signaling by interacting with erbB3 and erbB4 in glioblastoma cell lines. Exp Cell Res 317: 1476-1489, 2011.

24. Baselga J and Swain SM: Novel anticancer targets: revisiting ERBB2 and discovering ERBB3. Nat Rev Cancer 9: 463-475, 2009.

25. Zhang Y, Opresko L, Shankaran H, Chrisler WB, Wiley HS and Resat H: HER/ErbB receptor interactions and signaling patterns in human mammary epithelial cells. BMC Cell Biol 10: 78, 2009.

26. Lee D, Yu M, Lee E, et al: Tumor-specific apoptosis caused by deletion of the ERBB3 pseudo-kinase in mouse intestinal epithelium. J Clin Invest 119: 2702-2713, 2009.

27. Vivanco I and Sawyers CL: The phosphatidylinositol 3-Kinase AKT pathway in human cancer. Nat Rev Cancer 2: 489-501, 2002.

28. Miller TW, Pérez-Torres M, Narasanna A, et al: Loss of Phosphatase and Tensin homologue deleted on chromosome 10 engages ErbB3 and insulin-like growth factor-I receptor signaling to promote antiestrogen resistance in breast cancer. Cancer Res 69: 4192-4201, 2009.

29. Lowenstein EJ, Daly RJ, Batzer AG, et al: The SH2 and SH3 domain-containing protein GRB2 links receptor tyrosine kinases to ras signaling. Cell 70: 431-442, 1992.

30. Batzer AG, Rotin D, Ureña JM, Skolnik EY and Schlessinger J: Hierarchy of binding sites for Grb2 and Shc on the epidermal growth factor receptor. Mol Cell Biol 14: 5192-5201, 1994.

31. Hallberg B, Rayter SI and Downward J: Interaction of Ras and Raf in intact mammalian cells upon extracellular stimulation. J Biol Chem 269: 3913-3916, 1994.

32. Liebmann C: Regulation of MAP kinase activity by peptide receptor signalling pathway: paradigms of multiplicity. Cell Signal 13: 777-785, 2001.

33. Pearson G, Robinson F, Beers Gibson T, et al: Mitogen-activated protein (MAP) kinase pathways: regulation and physiological functions. Endocr Rev 22: 153-183, 2001. 
34. Gridelli C, De Marinis F, Di Maio M, Cortinovis D, Cappuzzo F and Mok T: Gefitinib as first-line treatment for patients with advanced non-small-cell lung cancer with activating Epidermal Growth Factor Receptor mutation: implications for clinical practice and open issues. Lung Cancer 72: 3-8, 2011.

35. Tejani MA, Cohen RB and Mehra R: The contribution of cetuximab in the treatment of recurrent and/or metastatic head and neck cancer. Biologics 4: 173-185, 2010.

36. Lièvre A, Bachet JB, Boige V, et al: KRAS mutations as an independent prognostic factor in patients with advanced colorectal cancer treated with cetuximab. J Clin Oncol 26: 374-379, 2008.

37. Pirker R, Pereira JR, Szczesna A, et al: Cetuximab plus chemotherapy in patients with advanced non-small-cell lung cancer (FLEX): an open-label randomised phase III trial. Lancet 373 $1525-1531,2009$

38. Slamon DJ, Leyland-Jones B, Shak S, et al: Use of chemotherapy plus a monoclonal antibody against HER2 for metastatic breas cancer that overexpresses HER2. N Engl J Med 344: 783-792, 2001.

39. Bang YJ, Van Cutsem E, Feyereislova A, et al: Trastuzumab in combination with chemotherapy versus chemotherapy alone for treatment of HER2-positive advanced gastric or gastrooesophageal junction cancer (ToGA): a phase 3, open-label, randomised controlled trial. Lancet 376: 687-697, 2010.

40. Ji XD, Li G, Feng YX, et al: EphB3 is overexpressed in non-smallcell lung cancer and promotes tumor metastasis by enhancing cell survival and migration. Cancer Res 71: 1156-1166, 2011.

41. Holbro T, Beerli RR, Maurer F, Koziczak M, Barbas CF III, and Hynes NE: The ErbB2/ErbB3 heterodimer functions as an oncogenic unit: ErbB2 requires ErbB3 to drive breast tumor cell proliferation. Proc Natl Acad Sci USA 100: 8933-8938, 2003.

42. Smirnova T, Zhou ZN, Flinn RJ, et al: Phosphoinositide 3-kinase signaling is critical for ErbB3-driven breast cancer cell motility and metastasis. Oncogene 31: 706-715, 2012.

43. Sergina NV, Rausch M, Wang D, et al: Escape from HER-family tyrosine kinase inhibitor therapy by the kinase-inactive HER3. Nature 445: 437-441, 2007.

44. Erjala K, Sundvall M, Junttila TT, et al: Signaling via ErbB2 and ErbB3 associates with resistance and epidermal growth factor receptor (EGFR) amplification with sensitivity to EGFR inhibitor gefitinib in head and neck squamous cell carcinoma cells. Clin Cancer Res 12: 4103-4111, 2006.

45. Zhang Y, Linn D, Liu Z, et al: EBP1, an ErbB3-binding protein, is decreased in prostate cancer and implicated in hormone resistance. Mol Cancer Ther 7: 3176-3186, 2008.

46. Desbois-Mouthon C, Baron A, Blivet-Van Eggelpoël MJ, et al: Insulin-like growth factor-1 receptor inhibition induces a resistance mechanism via the epidermal growth factor receptor/HER3/AKT signaling pathway: rational basis for cotargeting insulin-like growth factor-1 receptor and epidermal growth factor receptor in hepatocellular carcinoma. Clin Cancer Res 15: 5445-5456, 2009.

47. Frogne T, Benjaminsen RV, Sonne-Hansen K, et al: Activation of ErbB3, EGFR and Erk is essential for growth of human breast cancer cell lines with acquired resistance to fulvestrant. Breast Cancer Res Treat 114: 263-275, 2009.

48. Engelman JA, Zejnullahu K, Mitsudomi T, et al: $M E T$ amplification leads to gefitinib resistance in lung cancer by activating ERBB3 signaling. Science 316: 1039-1043, 2007.

49. Grøvdal LM, Kim J, Holst MR, Knudsen SLJ, Grandal MV and van Deurs B: EGF receptor inhibitors increase ErbB3 mRNA and protein levels in breast cancer cells. Cell Signal 24: 296-301, 2012

50. McDonagh CF, Huhalov A, Harms BD, et al: Antitumor activity of a novel bispecific antibody that targets the ErbB2/ErbB3 oncogenic unit and inhibits heregulin-induced activation of ErbB3. Mol Cancer Ther 11: 582-593, 2012.

51. Sithanandam G, Fornwald LW, Fields JR, Morris NL and Anderson LM: Anti-tumor efficacy of naked siRNAs for ERBB3 or AKT2 against lung adenocarcinoma cell xenografts. Int J Cancer 130: 251-258, 2012.

52. Engelman JA, Jänne PA, Mermel C, et al: ErbB-3 mediates phosphoinositide 3-kinase activity in gefitinib-sensitive non-small cell lung cancer cell lines. Proc Natl Acad Sci USA 102: 3788-3793, 2005 .

53. Ogino A, Kitao H, Hirano S, et al: Emergence of epidermal growth factor receptor $\mathrm{T} 790 \mathrm{M}$ mutation during chronic exposure to gefitinib in a non-small cell lung cancer cell line. Cancer Res 67: 7807-7814, 2007.
54. Sequist LV, Waltman BA, Dias-Santagata D, et al: Genotypic and histological evolution of lung cancers acquiring resistance to EGFR inhibitors. Sci Transl Med 3: 75ra26, 2011.

55. Kwak E: The role of irreversible HER family inhibition in the treatment of patients with non-small cell lung cancer. Oncologist 16: 1498-1507, 2011.

56. Ling B, Wang GX, Long G, Qiu JH and Hu ZL: Tumor suppressor miR-22 suppresses lung cancer cell progression through posttranscriptional regulation of ErbB3. J Cancer Res Clin Oncol 138: 1355-1361, 2012.

57. Kawano O, Sasaki H, Endo K, et al: ErbB3 mRNA expression correlated with specific clinicopathologic features of Japanese lung cancers. J Surg Res 146: 43-48, 2008.

58. Vaught DB, Stanford JC, Young C, et al: HER3 is required for HER2-induced preneoplastic changes to the breast epithelium and tumor formation. Cancer Res 72: 2672-2682, 2012.

59. Lee-Hoeflich ST, Crocker L, Yao E, et al: A central role for HER3 in HER2-amplified breast cancer: implications for targeted therapy. Cancer Res 68: 5878-5887, 2008.

60. Agus DB, Akita RW, Fox WD, et al: Targeting ligand-activated ErbB2 signaling inhibits breast and prostate tumor growth. Cancer Cell 2: 127-137, 2002.

61. Göstring L, Malm M, Höidén-Guthenberg I, et al: Cellular effects of HER3-specific affibody molecules. PLoS One 7: e40023, 2012.

62. Weiner DB, Kokai Y, Wada T, Cohen JA, Williams WV and Greene MI: Linkage of tyrosine kinase activity with transforming ability of the p185neu oncoprotein. Oncogene 4: 1175-1183, 1989.

63. Menendez JA and Lupu R: Transphosphorylation of kinase-dead HER3 and breast cancer progression: a new standpoint or an old concept revisited? Breast Cancer Res 9: 111, 2007.

64. Iorio MV and Croce CM: MicroRNAs in cancer: small molecules with a huge impact. J Clin Oncol 27: 5848-5856, 2009.

65. Yao E, Zhou W, Lee-Hoeflich ST, et al: Suppression of HER2/HER3-mediated growth of breast cancer cells with combinations of GDC-0941 PI3K inhibitor, trastuzumab, and pertuzumab. Clin Cancer Res 15: 4147-4156, 2009.

66. Junttila TT, Akita RW, Parsons K, et al: Ligand-independent HER2/HER3/PI3K complex is disrupted by trastuzumab and is effectively inhibited by the PI3K inhibitor GDC-0941. Cancer Cell 15: 429-440, 2009.

67. Chakrabarty A, Sánchez V, Kuba MG, Rinehart C and Arteaga CL: Feedback upregulation of HER3 (ErbB3) expression and activity attenuates antitumor effect of PI3K inhibitors. Proc Natl Acad Sci USA 109: 2718-2723, 2012.

68. Kim JH, Im KS, Kim NH, Yhee JY, Nho WG and Sur JH: Expression of HER-2 and nuclear localization of HER-3 protein in canine mammary tumors: histopathological and immunohistochemical study. Vet J 189: 318-322, 2011.

69. Chiu CG, Masoudi H, Leung S, et al: HER-3 overexpression is prognostic of reduced breast cancer survival: a study of 4046 patients. Ann Surg 251: 1107-1116, 2010.

70. Giltnane JM, Moeder CB, Camp RL and Rimm DL: Quantitative multiplexed analysis of ErbB family coexpression for primary breast cancer prognosis in a large retrospective cohort. Cancer 115: 2400-2409, 2009.

71. Haas S, Gevensleben H, Rabstein S, et al: Expression of heregulin, phosphorylated HER-2, HER-3 and HER-4 in HER-2 negative breast cancers. Oncol Rep 21: 299-304, 2009.

72. Gori S, Foglietta J, Mameli MG, et al: HER-3 status by immunohistochemistry in HER-2-positive metastatic breast cancer patients treated with trastuzumab: correlation with clinical outcome. Tumori 98: 39-44, 2012.

73. Yoshioka T, Nishikawa Y, Ito R, et al: Significance of integrin $\alpha v \beta 5$ and erbB3 in enhanced cell migration and liver metastasis of colon carcinomas stimulated by hepatocyte-derived heregulin. Cancer Sci 101: 2011-2018, 2010.

74. Scartozzi M, Mandolesi A, Giampieri R, et al: The role of HER-3 expression in the prediction of clinical outcome for advanced colorectal cancer patients receiving irinotecan and cetuximab. Oncologist 16: 53-60, 2011.

75. Zhang XL, Yang YS, Xu DP, et al: Comparative study on overexpression of HER $2 /$ neu and HER3 in gastric cancer. World $\mathbf{J}$ Surg 33: 2112-2118, 2009.

76. Zhu S, Belkhiri A and El-Rifai W: DARPP-32 increases interactions between epidermal growth factor receptor and ERBB3 to promote tumor resistance to gefitinib. Gastroenterology 141: $1738-1748,2011$.

77. Moelans CB, van Diest PJ, Milne AN and Offerhaus GJ: Her-2/neu testing and therapy in gastroesophageal adenocarcinoma. Patholog Res Int 2011: 674182, 2010. 
78. te Velde EA, Franke AC, van Hillegersberg R, et al: HER-family gene amplification and expression in resected pancreatic cancer. Eur J Surg Oncol 35: 1098-1104, 2009.

79. Hirakawa T, Nakata B, Amano R, et al: HER3 overexpression as an independent indicator of poor prognosis for patients with curatively resected pancreatic cancer. Oncology 81: 192-198, 2011.

80. Buck E, Eyzaguirre A, Haley JD, Gibson NW, Cagnoni P and Iwata KK: Inactivation of Akt by the epidermal growth factor receptor inhibitor erlotinib is mediated by HER-3 in pancreatic and colorectal tumor cell lines and contributes to erlotinib sensitivity. Mol Cancer Ther 5: 2051-2059, 2006.

81. Hsieh SY, He JR, Yu MC, et al: Secreted ERBB3 isoforms are serum markers for early hepatoma in patients with chronic hepatitis and cirrhosis. J Proteome Res 10: 4715-4724, 2011.

82. Blivet-Van Eggelpoël MJ, Chettouh H, Fartoux L, et al: Epidermal growth factor receptor and HER-3 restrict cell response to sorafenib in hepatocellular carcinoma cells. J Hepatol 57: 108-115, 2012.

83. Sheng Q, Liu X, Fleming E, et al: An activated ErbB3/NRG1 autocrine loop supports in vivo proliferation in ovarian cancer cells. Cancer Cell 17: 298-310, 2010.

84. Tanner B, Hasenclever D, Stern K, et al: ErbB-3 predicts survival in ovarian cancer. J Clin Oncol 24: 4317-4323, 2006.
85. Leng J, Lang J, Shen K and Guo L: Overexpression of p53, EGFR, c-erbB2 and c-erbB3 in endometrioid carcinoma of the ovary. Chin Med Sci J 12: 67-70, 1997.

86. Soler M, Mancini F, Meca-Cortes O, et al: HER3 is required for the maintenance of neuregulin-dependent and -independent attributes of malignant progression in prostate cancer cells. Int $\mathrm{J}$ Cancer 125: 2565-2575, 2009.

87. Buac K, Xu M, Cronin J, Weeraratna AT, Hewitt SM and Pavan WJ: NRG1/ERBB3 signaling in melanocyte development and melanoma: inhibition of differentiation and promotion of proliferation. Pigment Cell Melanoma Res 22: 773-784, 2009.

88. Ueno Y, Sakurai H, Tsunoda S, et al: Heregulin-induced activation of ErbB3 by EGFR tyrosine kinase activity promotes tumor growth and metastasis in melanoma cells. Int J Cancer 123: 340-347, 2008.

89. Belleudi F, Marra E, Mazzetta F, et al: Monoclonal antibodyinduced ErbB3 receptor internalization and degradation inhibits growth and migration of human melanoma cells. Cell Cycle 11: 1455-1467, 2012.

90. Djerf Severinsson EA, Trinks C, Gréen H, et al: The pan-ErbB receptor tyrosine kinase inhibitor canertinib promotes apoptosis of malignant melanoma in vitro and displays antitumor activity in vivo. Biochem Biophys Res Commun 414: 563-568, 2011. 